\title{
Brazilian Jiu-Jitsu and inhibitory control: effects of practice on secondary public school students in Abu Dhabi, UAE
}

\author{
Jean Carlo Benetti BUENO* ${ }^{* 1,2}$, Luísa SAAVEDRA ${ }^{1}$ \\ ${ }^{1}$ Fernando Pessoa University - UFP (Portugal) \\ ${ }^{2}$ Abu Dhabi Education Council - ADEC (United Arab Emirates)
}

\author{
5th IMACSSS World Scientific Congress Abstracts, Rio Maior (Portugal), October 6-8 \\ Section: Psychological, pedagogic and didactics in MA\&CS \\ Type: Poster communication
}

\section{Introduction}

Executive Functions (EF) are related to the amount of necessary and regulatory processes needed to maintain the focus, attention and resistance to external or internal distractions (Diamond, 2013). Through this process, it is possible to play with ideas, think before acting, as well as anticipate challenges by resistance to temptations and impulses. Since the 1950s, we have seen a progressive increase in studies involving physical activity, academic performance and cognition (Castelli, et al. 2015). Only more recently the Martial Arts (MA) become a greater interest to the scientific community due to its impact on human behavior. Faced with this, we have shown an increase in the number of school age children who have participated in MA training centers.

With great daring and futuristic vision of development and education, the United Arab Emirates (UAE) deployed in 2008 an educational program using Brazilian Jiu Jitsu - BJJ as a compulsory subject in public schools in Abu Dhabi (Al Housani, 2012). However, factors such as the wide range of MA, educational proposals, philosophies, sportization and Westernization of Middle Easterners, Gulf and scientific heterogeneity, as well as misconceptions about the cognitive effects of BJJ in children, has made the concept formation about the actual effects of BJJ on EF development, specifically on Inhibitory Control - IC complex. Thus, for clarification, this study aims to identify the effects of the practice of BJJ on the IC and to correlate the number of participants in BJJ classes and its effects.

\section{Methodology}

This study was conducted in a secondary public school in Abu Dhabi, UAE, using a sample of 156 boys ranging from 10 to 12-year-old students from grade 6 . All consecutive referrals were eligible except for children with diagnosed learning disability, or participated in extracurricular activities in addition to BJJ classes, or did not return a parental permission letter. The classes performed in 45 min sessions, 20\% for recreational purposes, 1 to 3 times per week for a period of 6 months. A Stroop Test model, Victoria version (Victoria Stroop Test - VST) was used (Troyer, et al., 2006). This study employed a quasi-experimental design with pre-post tests. Statistical analysis was performed using SPSS for Mac (version 32.0, Chicago, Ill). A t-test for paired samples was performed to compare pre - post results. A Multiple comparative POST HOC with Tukey test and HSD has been made to evaluate the differences between the levels of participation and the Stroop test. Pearson Test (r) was used to confirm the degrees of relationship between variables. The Linear Regression Test $\left(\mathrm{r}^{2}\right)$ was used to explain how the variation is common among elements and the Durbin-Watson Test $(\mathrm{dw})$ in order to exclude the hypothesis of serial autocorrelation was performed. Effect Size (ES) was calculated using a Cohen Model (d) with a qualitative assessment of ( 0.2 to $0.5=$ small, 0.5 to $0.8=$ medium and $>0.8=$ large) (Portney \& Watkins, 2009). 


\section{Results}

Of the 156 students evaluated, only 59 met the selection criteria. The results illustrated significant pre-post differences in reading tests of Colored Dots (D), reading Congruent Words (W), reading Incongruent Words (WC) and an Interference Control Score (IC) $\mathrm{p}<.05$, but a Medium ES was only found in reading incongruent words $d=.70$ and $d=.64$ INT score. The Good Participation (> 24 lessons) offered statistical differences and Large ES of the WC test compared to the Poor Participation (0-11 classes) p<.01, d=1.93 and Fair Participation (12-23 classes), $p>.01, d=1.17$ and IC compared to Poor Participation, $\mathrm{p}<.01, \mathrm{~d}=1.53$ and Fair Participation $\mathrm{p}<0.1, \mathrm{~d}=.93$. Participation illustrated strong evidence $\mathrm{p}<0.01$ and moderate correlation, free of autocorrelation, with WC Test, $\mathrm{r}=0.52, \mathrm{dw}=1.943$ and IC score, $\mathrm{r}=0.45, \mathrm{dw}=1.962$, as well as, evidenced an influence in $27.3 \%$ $\left(\mathrm{r}^{2}=0.27\right)$ the incongruent word speed reading WC and $20.8 \%\left(\mathrm{r}^{2}=0.20\right)$ the IC.

\section{Discussion and conclusion}

The results offered strong evidence that practicing BJJ had a positive influence on the Executive Functions development, specifically the Inhibitory Control - IC. Additionally, it was found that not only gains in cognitive ES were superior to average of other forms of Physical Activity (Castelli, et al., 2015.), but also showed similar ES compared to traditional martial arts (Kin, 2015; Douris, et al. 2015; Lakes \& Hoyt, 2004). Moreover, a moderate correlation in the participation illustrated an influence in $27.3 \%$ of the IC face to irrelevant activities, and $20.0 \%$ face to automatic activities. For all these aspects, our study showed evidence that may be relevant to MA coaches, teachers, psychologists, medical doctors, principals and managers linked to the educational and cognitive context in a way to better understand the participation of BJJ in social, educational, academic and scientific projects and activities. Finally, our study suggests that the practice of BJJ is positively correlated with self-control, focus, reading speed and decision-making, as well as being an excellent aid tool in favor of academical performance, mental health, social behavior and professional success of Emirati youth.

\section{References}

Al Housani, M. (2012, March 6) Royal vision that led to growth of Brazilian jiu-jitsu in Abu Dhabi. THE NATIONAL, April, 23 Retrieved from: http://www.thenational.ae/sport/other-sport/royalvision-that-led-to-growth-of-brazilian-jiu-jitsu-in-abu-dhabi\#page1

Castelli, D., Centeio, E., Hwang, J., Barcelona, J.M., Glowacki, E.M., Calvert, H.G., \& Nicksic, H.M. (2014). VIII. The History of Physical Activity and Academic Performance Research: Informing The Future. Monographs of the Society for Research in Child Development, 79, 119-148.

Diamond, A. (2013). Executive Functions. Annual review of psychology, 64, 135-168

Douris, P., Douris, C., Nicole, B. (2015) Martial Art Training and Cognitive Performance in MiddleAged Adults. Journal of Human Kinetics, 47, 277-283

Kim, Y. (2015) The effect of regular Taekwondo exercise on Brain-derived neurotrophic factor and Stroop test in undergraduate student. Journal of exercise nutrition \& biochemistry, 19(2), 73-79

Lakes, K., \& Hoyt, W. (2004). Promoting self-regulation through school-based martial arts training. Applied Developmental Psychology, 25(3), 283-302

Portney, L., \& Watkins, M. (2009). Foundations of clinical research: Applications to practice (3 ${ }^{\text {rd }}$ ed.). Upper Saddle River, NJ: Pearson Prentice Hall.

Troyer, A., Leach, L., \& Strauss, S. (2006). Aging and response inhibition: Normative data for the Victoria Stroop Test. Aging, Neuropsychology, and Cognition, 13(1), 20-35.

Key words: Brazilian Jiu-Jitsu; BBJ; executive functions; interference control; Emirati youth; combat sports.

This study has beensupported by Abu Dhabi Education Council - ADEC - UAE. 